\title{
3-Regular Non 3-Edge-Colorable Graphs with Polyhedral Embeddings in Orientable Surfaces ${ }^{\star}$
}

\author{
Martin Kochol \\ MÚ SAV, Štefánikova 49, 81473 Bratislava 1, Slovakia and FPV ŽU \\ kochol@savba.sk
}

\begin{abstract}
The Four Color Theorem is equivalent with its dual form stating that each 2-edge-connected 3-regular planar graph is 3-edgecolorable. In 1968, Grünbaum conjectured that similar property holds true for any orientable surface, namely that each 3-regular graph with a polyhedral embedding in an orientable surface has a 3 -edge-coloring. Note that an embedding of a graph in a surface is called polyhedral if its geometric dual has no multiple edges and loops. We present a negative solution of this conjecture, showing that for each orientable surface of genus at least 5, there exists a 3-regular non 3-edge-colorable graph with a polyhedral embedding in the surface.
\end{abstract}

\section{Introduction}

Edge-coloring of cubic (3-regular) graphs is an important topic in graph theory and theoretical computer science. By Tait [11, a cubic planar graph is 3-edgecolorable if an only if its geometric dual is 4-colorable. Since geometric dual of a 2-edge-connected planar cubic graph is a planar triangulation, the Four Color Theorem (see [2]) is equivalent to the statement that every 2-edge-connected planar cubic graph has a 3-edge-coloring.

Nonplanar cubic graphs do not need to be 3-edge-colorable. The best know example is the Petersen graph (see Fig. 10. In fact, by Holyer [8, the problem to decide whether a cubic graph is 3-edge-colorable is NP-complete.

An embedding of a graph in a surface is called polyhedral if its dual has no multiple edges and loops. In 1968, Grünbaum [7] presented a conjecture that each 3-regular graph with a polyhedral embedding in an orientable surface has a 3-edge-coloring. If this is true, it would generalize the dual form of the Four Color Theorem for any orientable surface.

In this paper we disprove the Grünbaum's conjecture and for every orientable surface of genus at least 5, we construct non 3-edge-colorable cubic graphs with a polyhedral embedding in the surface.

Note that Petersen graph has a polyhedral embedding in projective plane. Thus Grünbaum's conjecture has a sense only for orientable surfaces. More details about this conjecture and related results can be found in [13412. Basic facts about embeddings of graphs into surfaces can be found in [5]6].

^ Supported by grant VEGA 2/7037/7 and by A. v. Humboldt Fellowship. 


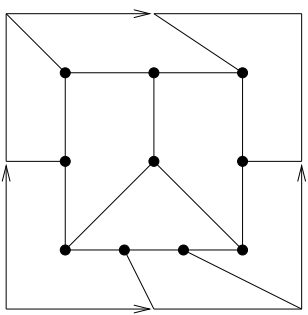

(a)

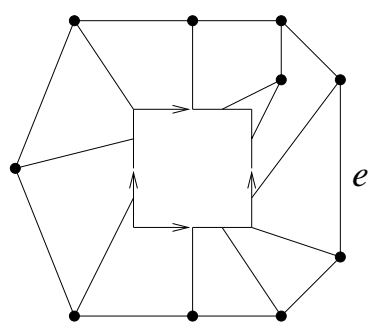

(b)

Fig. 1.

\section{Snarks and Superposition}

By a snark we mean a cubic graph without a 3-edge-coloring. It is well known (see, e.g., 10]) that any cubic graph with a bridge (1-edge-cut) is a snark. Such snarks are considered to be trivial. A nontrivial snark is the Petersen graph (see Fig. 11).

Suppose $v$ is a vertex of a graph $G$. Let $G^{\prime}$ arise from $G$ in the following process. Replace $v$ by a graph $H_{v}$ so that each edge e of $H$ having one end $v$ has one end from $H_{v}$. If $e$ is a loop having both ends $v$, then both ends of $e$ become vertices of $H_{v}$. Then $G^{\prime}$ is called v-superposition or a vertex superposition of $G$.

Suppose $e$ is an edge of $G$ with ends $u$ and $v$. Let $G^{\prime}$ arises from $G$ in the following process. Replace $e$ by a graph $H_{e}$ having at least two vertices, i.e., we delete $e$, pick up two distinct vertices $u^{\prime}, v^{\prime}$ of $H_{e}$ and identify $u^{\prime}$ with $u$ and $v^{\prime}$ with $v$. Then $G^{\prime}$ is called an e-superposition or an edge superposition of $G$. Furthermore, if $H_{e}$ is a snark, then $G^{\prime}$ is called a strong e-superposition or a strong edge superposition of $G$.

We say that a graph $G^{\prime}$ is a (strong) superposition of $G$ if $G^{\prime}$ arises from $G$ after finitely many vertex and (strong) edge superpositions. The following statement was proved in [10, Lemma 4.4] (see [910] for more details).

Lemma 1. Let $G$ be a snark and $G^{\prime}$ be a strong superposition of $G$. Furthermore, suppose that $G^{\prime}$ is cubic. Then $G^{\prime}$ is a snark.

\section{Constructions}

Clearly, a graph has an embedding in an orientable surface of genus $n$ if and only if it has an embedding in the plane with $n$ handles. In parts (a) and (b) of Fig. 1 are embeddings of the Petersen graph in the torus and in the plane with one handle, respectively. (If we identify the opposite segments of the square in part (b) of Fig. 1, we get a handle on the plane.)

Replacing edge $e$ by another copy of Petersen graph we get graph $G_{18}$ from Fig. 2. Replacing in $G_{18}$ the vertices of degree 5 by paths of length 2 , we get a 


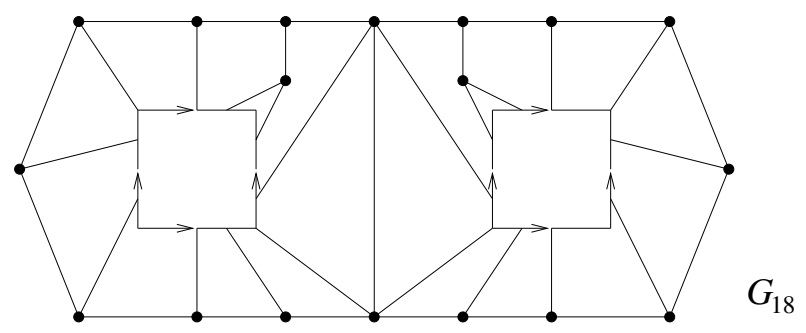

Fig. 2 .

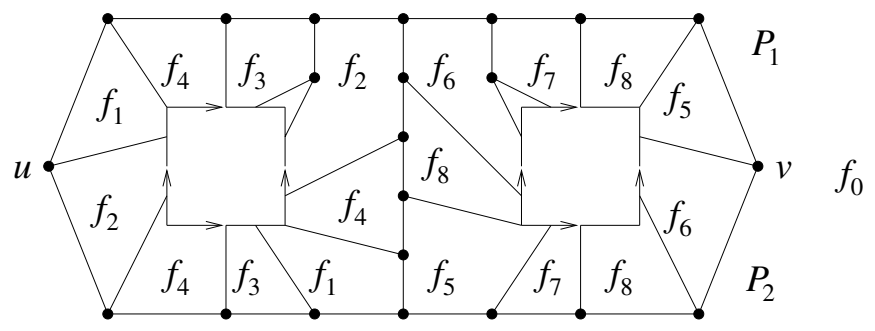

Fig. 3.

cubic graph $G$ indicated in Fig. 3. By Lemma 1, $G$ is a snark. The boundary of the infinite face $f_{0}$ is a circuit, composed from paths $P_{1}$ and $P_{2}$ with ends $u$ and $v$. The following holds true.

(1) any two faces $f_{i}, f_{j}, i, j \in\{1, \ldots, 8\}$, share at most one edge,

(2) the infinite face $f_{0}$ share exactly two edges with each $f_{i}, i \in\{1, \ldots, 8\}$ so that $P_{1}$ and $P_{2}$ contain exactly one of them.

Properties (1) and (2) are important. We can take a nonpolyhedral embedding of a snark in an orientable surface, and replacing some of its edges by copies of $G$ and some vertices by suitable graphs, we can get snarks with polyhedral embeddings in orientable surface. By replacing an edge of a copy of $G$, we identify the ends of $e$ by $u$ and $v$, respectively. For example, in Fig. 4is a snark constructed in [4, Fig. 8]. Let us note that this is not a polyhedral embedding in the torus, because the pairs of faces $a_{1}, a_{2}$ and $b_{1}, b_{2}$ have two edges in common (i.e., its geometric dual has two pairs of parallel edges). In order to remove this obstacle, we replace edges $e_{1}$ and $e_{2}$ by two copies of $G$ and we get graph $G_{66}$ indicated in Fig. 5. Replacing in $G_{66}$ the vertices of degree 5 by paths of length 2 we get the graph indicated in Fig. 6. By Lemma 1, this is a snark. Furthermore, by (1) and (2), any two faces of this graph have at most one edge in common (i.e., the pairs of faces $a_{1}, a_{2}$ and $b_{1}, b_{2}$ are "separated" by the copies of graph $G$ ). Thus the geometric dual has no parallel edges and loops, i.e., we have a polyhedral embedding of a snark in orientable surface of genus 5 . 


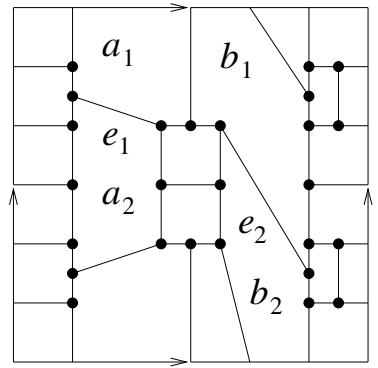

Fig. 4.

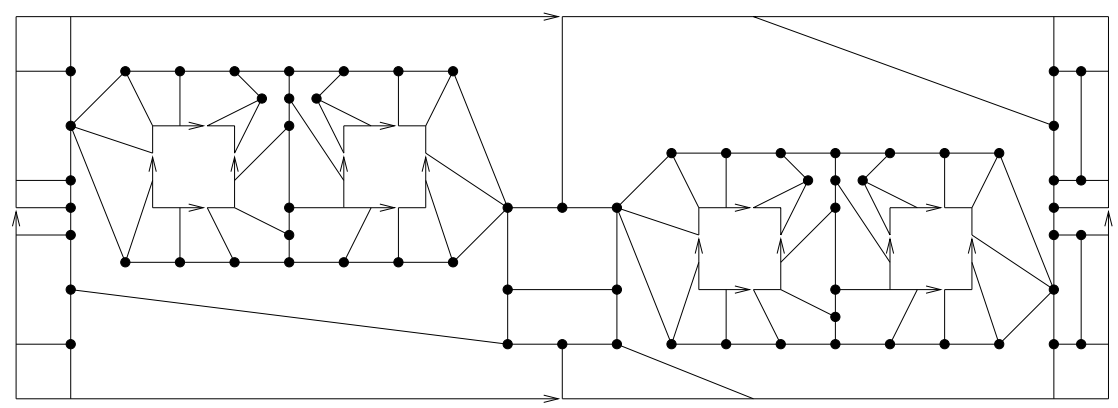

$G_{66}$

Fig. 5.

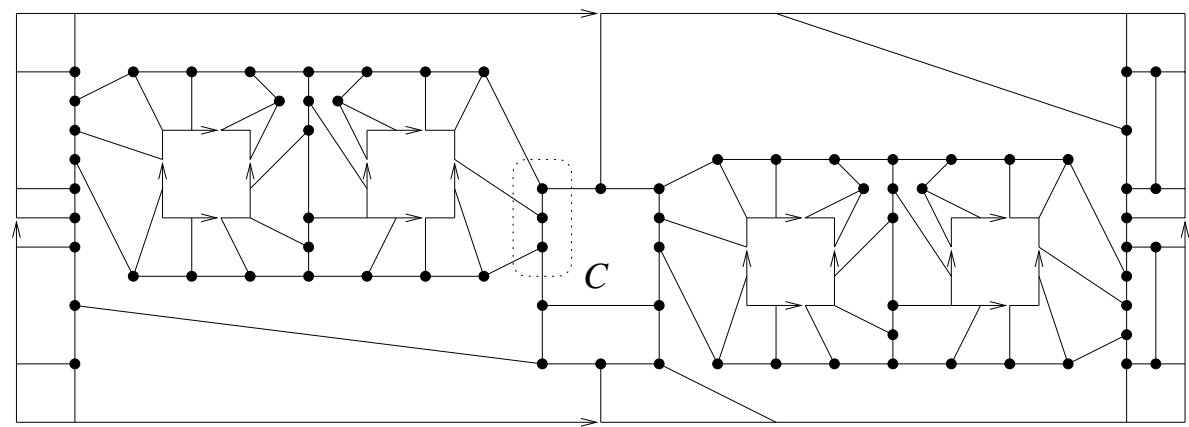

Fig. 6.

In order to get snarks with embeddings in orientable surfaces of genus $>5$, it suffices to replace a vertex of degree 5 from $G_{66}$ by suitable graphs with embeddings in a plane with handles. For example, consider the three vertices of the graph from Fig. 6 contained inside of the disc $C$ indicated by dotted line. Replacing them by the graph indicated in Fig. 7, we get a snark with polyhedral 


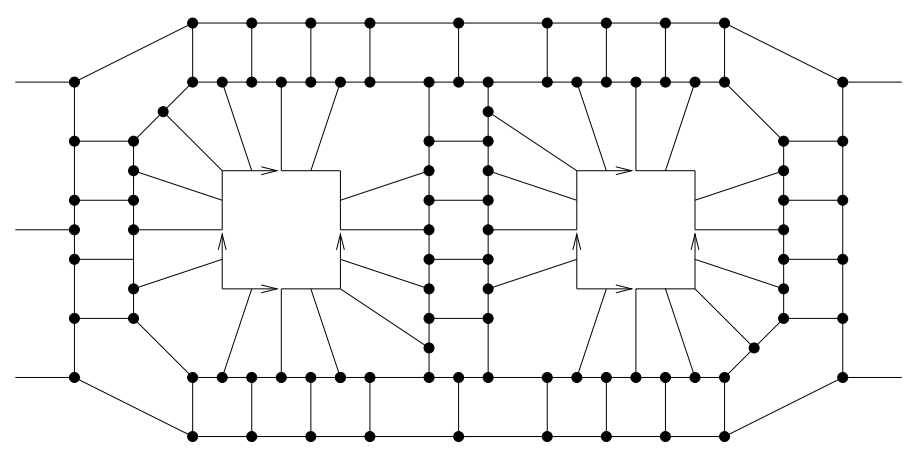

Fig. 7 .

embedding in surface of genus 7 . This snarks is a strong superposition of the snark from Fig. 4. In this way we can prove the following statement.

Theorem 1. For any orientable surface of genus $\geq 5$, there exists a 3-regular non-3-edge-colorable graph with a polyhedral embedding in this surface.

\section{References}

1. Albertson, M.O., Alpert, H., Belcastro, S.-M., Haas, R.: Grünbaum colorings of toroidal triangulations (manuscript) (April 2008)

2. Appel, K., Haken, W.: Every Planar Map Is Four Colorable. Contemp. Math., vol. 98. Amer. Math. Soc., Providence, RI (1989)

3. Archdeacon, D.: Problems in topological graph theory: Three-edge-coloring planar triangulations, http://www.emba.uvm.edu/ archdeac/problems/grunbaum.htm

4. Belcastro, S.-M., Kaminski, J.: Families of dot-product snarks on orientable surfaces of low genus. Graphs Combin. 23, 229-240 (2007)

5. Diestel, R.: Graph Theory, 3rd edn. Springer, Heidelberg (2005)

6. Gross, J.L., Tuker, T.W.: Topological Graph Theory. Wiley, New York (1987)

7. Grünbaum, B.: Conjecture 6. In: Tutte, W.T. (ed.) Recent Progress in Combinatorics, Proceedings of the Third Waterloo Conference on Combinatorics, May 1968, p. 343. Academic Press, New York (1969)

8. Holyer, I.: The NP-completeness of edge-coloring. SIAM J. Comput. 10, 718-720 (1981)

9. Kochol, M.: Snarks without small cycles. J. Combin. Theory Ser. B 67, 34-47 (1996)

10. Kochol, M.: Superposition and constructions of graphs without nowhere-zero $k$ flows. European J. Combin. 23, 281-306 (2002)

11. Tait, P.G.: Remarks on the colouring of maps. Proc. Roy. Soc. Edinburgh 10, 729 (1880)

12. Vodopivec, A.: On embedding of snarks in the torus. Discrete Math. 308, 1847-1849 (2008) 